\title{
Alpha decay rate enhancement in metals: An unlikely scenario
}

\author{
Nikolaj Thomas Zinner ${ }^{1,2, *}$ \\ ${ }^{1}$ Institute for Physics and Astronomy, University of Århus, DK-8000 Arhus C, Denmark \\ ${ }^{2}$ Gesellschaft für Schwerionenforschung GSI, Darmstadt, Germany
}

(Dated: August 22, 2018)

\begin{abstract}
It has been recently suggested that one might drastically shorten the alpha lifetime of nuclear waste products, if these are embedded in metals at low temperatures. Using quantum mechanical tunneling arguments, we show that such an effect is likely to be very small, if present at all.
\end{abstract}

PACS numbers: 25.30.Pt,25.85.-w,26.30.+k,95.30.Cq,97.60.-s,97.60.Bw

\section{INTRODUCTION}

In a recent publication, Kettner et. al. 1] have suggested that one could speed up alpha decay of transuranic nuclear waste material by embedding it in metals at low temperature. This would have a huge impact on waste disposal management and quite likely initiate a completely renewed public debate on the subject of nuclear energy. The proposed idea is that the electron screening provided by the metal will lower the alpha-decay lifetime. Perturbations to nuclear alpha decay energies and barriers is a basic problem that was clarified and solved many years ago [2]. The influence of an electron plasma environment on decay rates has also been carefully studied many times before and, as the present paper will demonstrate, the recently proposed effect is unlikely from the viewpoint of standard screening models.

\section{TUNNELING AND SCREENING}

The quantum mechanics of tunnelling is described in virtually all standard textbooks (see for instance [3]). The case of alpha decay goes back to Gamow who employed the WKB method to calculate the transition probability for an alpha particle to escape the coulomb barrier. In Fig. 1 we show a schematic illustration of the process, showing the nuclear potential (shown as a well inside $10 \mathrm{fm}$ ) and various barriers on a log scale. The radius describes the relative distance between the daughter nucleus (with charge $Z-2$ ) and the alpha-particle. The full line shows the $\alpha$ particle energy without any screening and the dots indicate the path that the particle has to tunnel through. If we embed the radioactive nuclei in a metal containing a density of free electrons in-medium screening corrections have to be considered as well. These in-medium effects can be described by an attractive screening potential (dashed line), whose magnitude on general grounds decreases with distance from the nucleus with a typical screening length scale $R$. (The amplitude of the screening potential is exaggerated for visual purposes.) The screening potential also affects the alpha-decay energy which will be lowered. If we assume that the screening potential is constant over the dimensions of the nucleus (this applies to the cases discussed here, as we will show below), then in a very good approximation the alpha-decay energy is lowered by the value of the constant screening potential at small distances (denoted by $\Delta U$, which is negative and is often called the screening energy [4]). Hence in the medium the alpha decay occurs with a shifted energy $E_{\alpha}+\Delta U$ (shown by the dashed line) rather than $E_{\alpha}$. (We note that this lowering of the alpha-decay energy has been neglected in [1], and also in [5] who also predicts a lowering of in-medium nuclear decay lifetimes). In the medium, the alpha decay proceeds through an effective barrier which is given by the sum of the Coulomb barrier and the screening potential; the effective barrier is lower than the one in the non-screened case. As Fig. 10 shows the path through the screened barrier will actually be longer than through the unscreened barrier, provided the screening potential varies over the distances of the tunnel process. As a consequence one expects a longer alpha-decay lifetime in the medium. Here we have assumed that the decay energy and screening energy is simply additive. In general the energy shift will depend on the screening potential. However, for the weak screening cases to be studied here our assumption is justified. In fact it has been experimentally verified in [1] which find that nuclear resonance energies are lowered by the screening energy if the target nuclei are embedded in a metallic medium. We also remark that in the extreme limit of sufficiently large screening (e.g. at very high densities), the alpha-decay lifetime becomes infinite

*Electronic address: zinner@phys.au.dk 
as the decaying state is shifted energetically below the decay threshold in the medium. Such a behavior is expected for example for pure alpha matter which will show a phase transition to ${ }^{8} \mathrm{Be}$ matter at densities in excess of a few $10^{9} \mathrm{~g} / \mathrm{cm}^{3}[6,7]$.

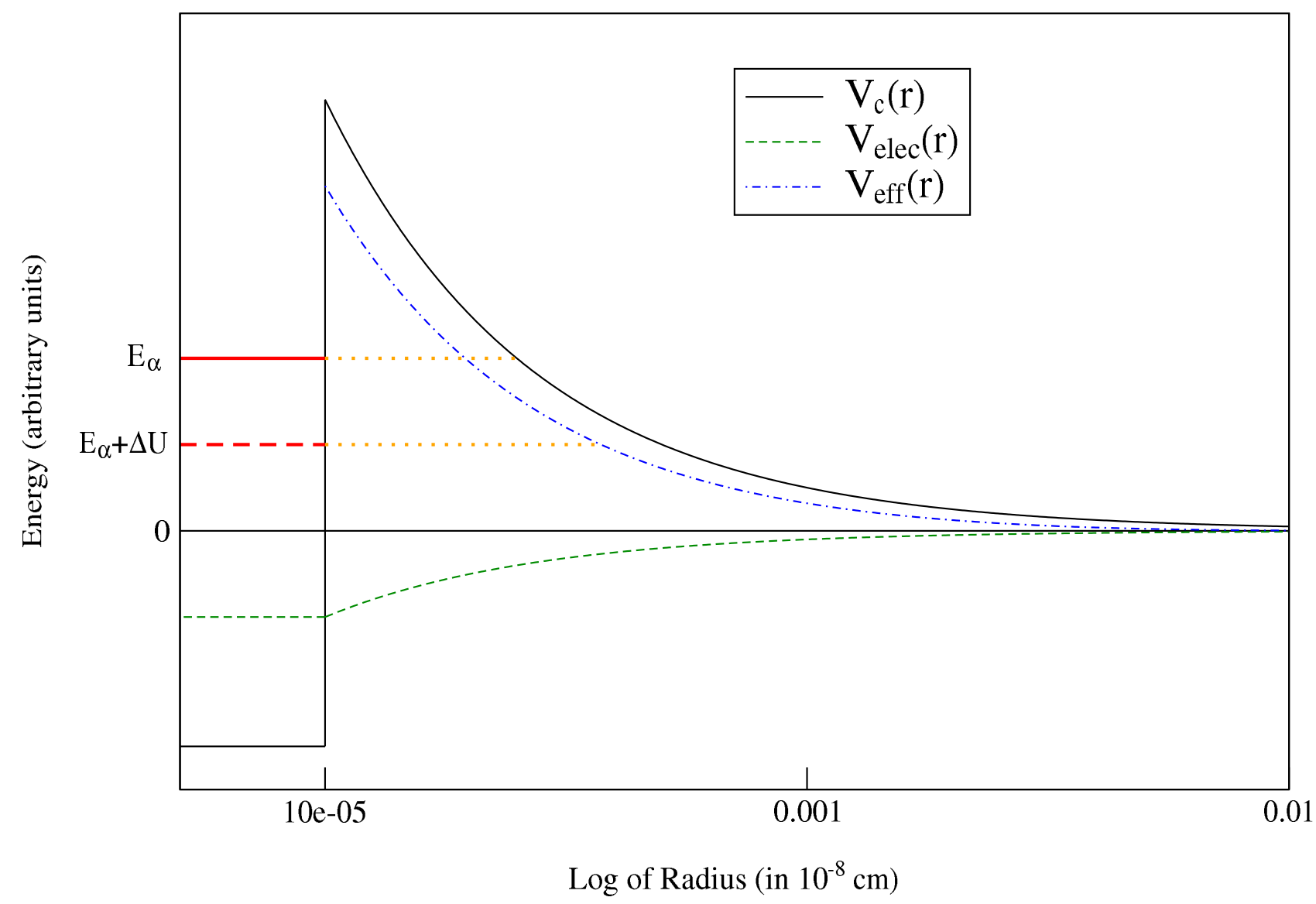

FIG. 1: Figure showing the basics of alpha tunneling. Shown are the pure coulomb barrier (full), the potential from the electron screening (dashed) and the effective screened potential (dash-dotted). The $\alpha$ particle energy with and without screening correction are shown inside the nuclear potential well. Also show are the tunneling paths. Notice that the radius is plotted on a $\log$ scale.

\section{BASIC MODELS AND RESULTS}

On the basis of the Debye scrrening model, it is implied in [1] that alpha-decay for ${ }^{210} \mathrm{Po}$ and ${ }^{226} \mathrm{Ra}$ can be significantly reduced if the decay occurs in a metal at low temperatures. For ${ }^{210}$ Po the experimental observation of such a reduction has already been reported and is ascribed to Debye screening [8]. We will use the two nuclei ${ }^{210}$ Po and ${ }^{226} \mathrm{Ra}$ as examples to demonstrate that standard screening models predict very small changes of the lifetimes if the nuclei are embedded in metals.

In the classical work on screening of nuclear reactions for astrophysical applications [9] the Debye-Hückel model was used for weak screening, with different corrections for electron degeneracy etc. Later works, including [10, 11], treated strong screening and also focused on different densities and temperatures. In the current case we are interested in the screening effect of electrons in metals at low temperature, which is a degenerate system. We therefore choose to work with the Thomas-Fermi model, which is basically the free electron gas model with additional assumptions on the chemical potential (see 12] for details). But we will also estimate the screening effects on the alpha lifetimes within the Debye model.

In both models (Thomas-Fermi and Debye) the Coulomb potential between two charges $Z_{1}, Z_{2}$ has to be replaced 
by an effective potential of Yukawa type $V_{\text {eff }}$ if medium effects are accounted for:

$$
V_{\text {eff }}(r)=\frac{Z_{1} Z_{2} e^{2}}{r} \exp (-r / R)=\frac{Z_{1} Z_{2} e^{2}}{r}+V_{s c}(r)
$$

where $V_{s c}$ is the in-medium screening potential. In the Thomas-Fermi (TF) model, the screening length scale (screening radius) is given by

$$
R_{T F}=\sqrt{\frac{\epsilon_{0} E_{F}}{3 e^{2} \rho}}
$$

Here $\rho$ is the electron density and $E_{F}$ the fermi energy. In natural units for metals one has

$$
R_{T F}=3.7 \cdot 10^{-10} \sqrt{\frac{E_{F}[e V]}{\rho\left[10^{22} \mathrm{~cm}^{-3}\right]}} \mathrm{cm}
$$

Using typical values $\left(\rho \sim 10 \cdot 10^{22} \mathrm{~cm}^{-3}\right.$ and $\left.E_{F} \sim 10 \mathrm{eV}[12]\right)$, one finds $R_{T F}=3.7 \cdot 10^{3}$ fm, which is more than two orders of magnitude larger than the nuclear dimensions. The screening potential should therefore not vary over the nuclear range, supporting our assumption of a constant value $\Delta U$ of the screening potential in the nuclear interior, by which the screening energy will be shifted. ${ }^{1}$

In the Debye-Hückel approximation [9], the screening radius $R_{D}$ is temperature dependent. If one puts in typical numbers for metals one finds

$$
R_{D}=2.18 \cdot 10^{-8} \sqrt{T[K]} \mathrm{cm}
$$

Assuming a temperature of 4 Kelvin [1], $R_{D}$ is two orders of magnitude larger than $R_{T F}$, again justifying our assumption of a constant screening potential inside the nucleus. However, the metal environment with its degeneracy requires modifications to the Debye-Hückel model (see [9, 11]), so the comparison should be regarded with some caution.

The alpha-decay halflife is dominated by the barrier penetration probability $P$ which one can evaluate within WKB theory, resulting in

$$
P \sim \exp (-2 S(E))
$$

where $S(E)$ is the action integral. For a non-screened Coulomb barrier one has

$$
S(E)=\sqrt{\frac{2 m}{\hbar^{2}}} \int_{R_{\mathrm{nuc}}}^{R_{0}} \sqrt{\left(\frac{e^{2} Z_{1} Z_{2}}{r}-E\right)} d r
$$

where $R_{\text {nuc }}$ is the nuclear radius (we will use $R_{\text {nuc }}=1.22 \cdot A^{1 / 3} \mathrm{fm}$, where $A$ is the nuclear mass number) and $R_{0}$ is the outer turning point. In the case of alpha decay one uses $Z_{1}=2$ and $Z_{2}=Z_{n u c}$, where $Z=Z_{1}+Z_{2}$ is the charge number of the decaying nucleus. Then the outer turning point is $R_{0}=2 Z_{\mathrm{nuc}} e^{2} / E$. In the presence of the screening potential the action becomes

$$
S_{s c}(E)=\sqrt{\frac{2 m}{\hbar^{2}}} \int_{R_{\mathrm{nuc}}}^{R_{1}} \sqrt{\left(V_{\mathrm{eff}}(r)-(E+\Delta U)\right)} d r=\sqrt{\frac{2 m}{\hbar^{2}}} \int_{R_{\mathrm{nuc}}}^{R_{1}} \sqrt{\left(\frac{Z_{1} Z_{2} e^{2}}{r}+V_{\mathrm{sc}}(r)-(E+\Delta U)\right)} d r
$$

where $R_{1}$ is the outer turning point for the screened barrier (see Fig. 11). As discussed above the alpha-decay energy has to be replaced by the shifted value $E+\Delta U$. We fix the value of $\Delta U$ by assuming that $V_{\mathrm{sc}}$ is constant over the nuclear dimensions, i.e. $V_{\mathrm{sc}}(0)=\Delta U=e^{2} Z_{1} Z_{2} / R$, were $R$ is the screening radius. If $V_{\mathrm{sc}}(r)$ is even constant for radii $r \leq R_{1}$, then the in-medium effects cancel and the alpha lifetime remains unchanged. Obviously modifications of the lifetime can be expected, if the screening potential varies over the radius scale of the outer turning point (e.g. at high densities were the screening length scale $R$ is small) or the alpha-decay energy is small. Estimating $R_{0}$ for

\footnotetext{
${ }^{1}$ Due to the singularity at $r=0$, neither the bare Coulomb nor the effective Yukawa potential can be the realistic solution at small nuclear radii. To avoid problems, we use a small constant nuclear radius as the inner turning point of the barrier.
} 
a typical alpha decay energy of $E=5 \mathrm{MeV}$, one finds $R_{0} \approx 51 \mathrm{fm}$ (for $Z=90$ ), which is much smaller than the Thomas-Fermi or Debye screening radii estimated above. Hence changes of the alpha decay lifetimes should be quite small. To estimate such changes we define the ratio between the halflives in the screened $T_{\alpha, s c}$ and unscreened case $T_{\alpha}$ :

$$
\mathcal{R}=\frac{T_{\alpha, s c}}{T_{\alpha}}=\frac{P}{P_{\mathrm{sc}}}
$$

with the penetration factors for screened and unscreened case as defined above. We have estimated this ratio for typical alpha-decay energies and various screening radii by calculating the action integrals (6) and (7) numerically. The results are summarized in Table \ As one can clearly see the effects are quite small for typical screening radii in metals (a few $10^{3} \mathrm{fm}$ in the Thomas-Fermi model). Moreover, Thomas-Fermi and Debye screening models both predict that the alpha-decay halflife should increase if the nucleus is embedded in a metal. If application of the Debye model is allowed to describe the in-medium screening, the screening length scale depends on temperature. But we find that even at temperatures of order Kelvin, the changes of the halflives should be very small. Thus our results agree with the findings of Ref. [13] which predicts that changes of alpha lifetimes due to electronic screening effects should be negligibly small (see also [2]).

As expected on general grounds, the in-medium modifications increase the lower the alpha decay energy (which implies a longer path under the barrier) and the smaller the screening radius (allowing for changes of the screening potential over the length of the tunnel path). The screening models predict a sizable enhancement once the screening radius gets of the same order as the outer turning point (of order a few times $10^{2} \mathrm{fm}$ ).

The alpha-decay energies of ${ }^{210} \mathrm{Po}$ and ${ }^{226} \mathrm{Ra}$ are $5.30 \mathrm{MeV}$ and $4.78 \mathrm{MeV}$, respectively. If we assume a typical screening length scale for metals of $R_{T F}=3.7 \cdot 10^{3} \mathrm{fm}$ (Thomas-Fermi model) or $R_{D}=4 \cdot 10^{5}$ fm $($ Debye model at 4 $\mathrm{K})$, we find that the alpha-decay lifetimes of these two nuclei should be very slightly enlarged if embedded in a metal. For ${ }^{210}$ Po we find $\mathcal{R}=1.009$ (Thomas-Fermi) and $\mathcal{R}=1.000$ (Debye), and for ${ }^{226}$ Ra $\mathcal{R}=1.013$ (Thomas-Fermi) and $\mathcal{R}=1.000$ (Debye). In turn, if experimentally a reduction of the ${ }^{210}$ Po halflife is observed in metals $[8]$, then the origin of this unexpected effect should not be due to in-medium screening as described by the Thomas-Fermi or Debye models.

In Ref. [1], the screening energy, which a ${ }^{210}$ Po nucleus experiences in a metal, is estimated as $\Delta U=420 \mathrm{keV}$, based on many studies of the observed screening enhancement of the $\mathrm{d}+\mathrm{d}$ fusion reaction cross section in metals 14, 15, 16]. This screening energy corresponds to a screening radius $R=562 \mathrm{fm}$ and using this value we find that the ${ }^{210}$ Po lifetime should be enhanced by $45 \%$ due to screening corrections based on the effective potential defined in (1),

\begin{tabular}{|l|c|c|c|c|c|c|}
\hline $\mathrm{E} / \mathrm{R}$ & $1.0 \cdot 10^{6}$ & $1.0 \cdot 10^{5}$ & $1.0 \cdot 10^{4}$ & $5.0 \cdot 10^{3}$ & $1.0 \cdot 10^{3}$ & $5.0 \cdot 10^{2}$ \\
\hline 3.0 & 1.000 & 1.000 & 1.006 & 1.025 & 1.828 & 10.95 \\
\hline 4.0 & 1.000 & 1.000 & 1.003 & 1.012 & 1.342 & 3.217 \\
\hline 5.0 & 1.000 & 1.000 & 1.002 & 1.007 & 1.184 & 1.954 \\
\hline 6.0 & 1.000 & 1.000 & 1.001 & 1.004 & 1.113 & 1.530 \\
\hline
\end{tabular}

TABLE I: Table of halflive ratios $\mathcal{R}$ as defined in equation (8) for various choices of the unscreened alpha-decay energies (in $\mathrm{MeV}$ ) and the in-medium screening radius (in fm). All calculations were done for nuclear charge $Z=90$ and mass $A=232$.

\section{CONCLUSION}

In summary, we have studied the in-medium effects on alpha lifetimes if the decaying nucleus is embedded in a metal. Based on standard screening models like the Thomas-Fermi model or the Debye approach we have given quite general arguments why the effects should be small and have then supported these by numerical calculations. Indeed we find negligible changes of the lifetimes for ${ }^{210} \mathrm{Po}$ and ${ }^{226} \mathrm{Ra}$, for which recently quite sizable effects have been predicted and reported [1, 8] based on applications of a Debye model for the host metal. Thus our calculations do not support the exciting idea that nuclear waste can be faster disposed of if embedded in metals at low temperatures due to significantly reduced lifetimes. We believe that the estimate given in 1], which has born out this idea, is incorrect as it does not account for the in-medium modification of the decay energy which, in first order, cancels the barrier reduction for the emitted alpha particle.

We like to remark that the influence of the medium for alpha decay is different than for other physical processes. It is wellknown that fusion reactions of charged particles are enhanced at low energies due to screening effects in the 
plasma [9] or in the laboratory [4, 17, 18]. This comes about as the relative energy of the fusing nuclei at the outer turning point, where the penetration process starts, is larger than the asymptotic energy due to screening effects. Or in an alternative picture, the fusing nuclei at a given energy have to penetrate through a barrier which is reduced by the screening effects. Of course, the medium also influences the alpha decay energy, i.e. the asymptotically measured energy in the medium is not the decay energy of the bare nuclear system, but has to be corrected for screening effects. However, such modifications reflect the variations of the screening potential mainly at radii larger than the outer turning point and hence do not change the decay halflives as the effective barrier, which the alpha particle has to penetrate, is nearly the same in the medium as in the bare nuclei. We mention that the medium modifications for alpha decay (or the decay by other charged particles) is similar to the resonant screening corrections in fusion reactions where particular care has to be taken for the corrections of the resonance energy [10, 19].

The medium corrections to alpha decay are also different to those for beta decay or electron captures. In both cases the weak-interaction nuclear matrix element is unchanged by the medium, however, phase space has to be corrected as parent and daughter nuclei have charge numbers which differ by one unit. Hence screening effects change the effective Q-value for beta decays or electron captures in the medium. Modifications of beta halflives due to screening effects in metals have for example been reported in 21. For electron captures one has additionally to consider that the capture rate is proportional to the electron probability at the nucleus which can be strongly changed by medium effects. A prominent example is ${ }^{7} \mathrm{Be}$, which as a bare nucleus in vacuum, has an infinite halflife, but in atoms decays by mainly K-shell electron capture [20]. In the sun, however, the ${ }^{7} \mathrm{Be}$ lifetime is noticeably increased as the electron probability at the nucleus is smaller in the solar plasma than in an atom [22]. Experimentally medium-modifications of the ${ }^{7} \mathrm{Be}$ lifetime have been reported in [23].

\section{Acknowledgments}

The author would like to thank Karlheinz Langanke for useful suggestions and invaluable discussion. Thanks goes also to Hans Feldmeier for helpful ideas and Jim Truran for pointing out several nice references. The author would like to thank GSI for its hospitality during which part of this work was done.

[1] K. U. Kettner et. al., J. Phys. G: Nucl. Part. Phys. 32 (2006) 489

[2] G.T. Emery, Ann. Rev. Nucl. Sci. 22 (1972) 165

[3] D. J. Griffiths: Introduction to Quantum Mechanics, 2nd Ed. Pearson Prentice Hall 2005

[4] H.J. Assenbaum, K. Langanke and C. Rolfs, Z. Phys. A327 (1987) 461

[5] T. Liolios, Phys. Rev. C 68 (2003) 015804

[6] K. Langanke, D. Lukas, H.-M. Müller, S. Schramm and S.E. Koonin, Z. Phys. A339 (1991) 419

[7] H.-M. Müller and K. Langanke, Phys. Rev. C49 (1994) 524

[8] C. Rolfs, as quoted in Frankfurter Allgemeine Zeitung 13.8 .2006 p. 56

[9] E. E. Salpeter, Australian J. Phys. 7 (1954) 373

[10] E. E. Salpeter and H. M. Van Horn, ApJ 155 (1969) 183

[11] H. E. Mitler, ApJ 212 (1977) 513

[12] N. W. Ashcroft and N. D. Mermin: Solid State Physics, Int. Ed. W.B. Saunders 1976

[13] W. Rubinson and M.L. Perlman, Phys. Lett. B40 (1972) 163

[14] F. Raiola it et al., Eur. Phys. J. A13 (2002) 377

[15] F. Raiola it et al., Eur. Phys. J. A19 (2004) 283

[16] F. Raiola it et al., Eur. Phys. J. A31 (2005) 1141

[17] S. Engstler et al., Phys. Lett. B202 (1988) 179

[18] F. Raiola el al.: Electron Screening: A Review, AIP Conference Proceedings - April 26, 2006 - Volume 831, pp. 296-303

[19] R. Cussons, K. Langanke and T. Liolios, Eur. Phys. J. A 15 (2002) 291

[20] C.E. Rolfs and W.S. Rodney, Cauldrons in the Cosmos, (University of Chicago Press, Chicago, 1988)

[21] B. Limata et al., Eur. Phys. J. A28 (2006) 251

[22] C.W. Johnson et al., Astr. J. 392 (1992) 320

[23] B. Wang et al., Eur. Phys. J. A28 (2006) 375 